\title{
In utero and peripubertal metals exposure in relation to reproductive hormones and sexual maturation and progression among boys in Mexico City
}

Pahriya Ashrap', John D. Meeker', Brisa N. Sánchez², Niladri Basu³, Marcela Tamayo-Ortiz ${ }^{4,5}$, Maritsa Solano-González ${ }^{4}$, Adriana Mercado-García ${ }^{4}$, Martha M. Téllez-Rojo ${ }^{4}$, Karen E. Peterson ${ }^{1,6}$ and Deborah J. Watkins ${ }^{1 *}$ (D)

\begin{abstract}
Background: Endocrine disrupting chemicals (EDCs) such as metals have been reported to alter circulating reproductive hormone concentrations and pubertal development in animals. However, the relationship has rarely been investigated among humans, with the exception of heavy metals, such as $\mathrm{Pb}$ and $\mathrm{Cd}$. Our aim was to investigate measures of in utero and peripubertal metal exposure in relation to reproductive hormone concentrations and sexual maturation and progression among boys from the Early Life Exposure in Mexico to Environmental Toxicants (ELEMENT) cohorts.

Methods: Our analysis included 118 pregnant women and their male children from the ELEMENT study. Essential and non-essential metals were measured in urine collected from the mothers during the third trimester of pregnancy and their male children at 8-14 years. Reproductive hormone concentrations [serum testosterone, estradiol, dehydroepiandrosterone sulfate (DHEA-S), inhibin B, and sex hormone-binding globulin (SHBG)] were measured in blood samples from the children at 8-14 years. We also assessed Tanner stages for sexual maturation (genital, pubic hair development, and testicular volume), at two time points (8-14, 10-18 years). We used linear regression to independently examine urinary metal concentrations in relation to each peripubertal reproductive hormones adjusting for child age and BMI. Generalized estimation equations (GEEs) were used to evaluate the association of in utero and peripubertal metal exposures with sexual maturation and progression during follow-up based on Tanner staging and testicular volume.

Results: In utero and prepubertal concentrations of some urinary metals were associated with increased concentrations of peripubertal reproductive hormones, especially non-essential metal(loid)s As and Cd (in utero), and Ba (peripubertal) as well as essential metal Mo (in utero) in association with testosterone. More advanced pubic hair developmental stage and higher testicular volume at the early teen visit was observed for boys with higher non-essential metal concentrations, including in utero Al and peripubertal Ba, and essential metal Zn concentration (peripubertal). These metals were also associated with slower pubertal progression between the two visits.

(Continued on next page)
\end{abstract}

\footnotetext{
* Correspondence: debjwat@umich.edu

'Department of Environmental Health Sciences, University of Michigan

School of Public Health, 1415 Washington Heights, Ann Arbor, Ml 48109,

USA

Full list of author information is available at the end of the article
}

(C) The Author(s). 2020 Open Access This article is licensed under a Creative Commons Attribution 4.0 International License, which permits use, sharing, adaptation, distribution and reproduction in any medium or format, as long as you give appropriate credit to the original author(s) and the source, provide a link to the Creative Commons licence, and indicate if changes were made. The images or other third party material in this article are included in the article's Creative Commons licence, unless indicated otherwise in a credit line to the material. If material is not included in the article's Creative Commons licence and your intended use is not permitted by statutory regulation or exceeds the permitted use, you will need to obtain permission directly from the copyright holder. To view a copy of this licence, visit http://creativecommons.org/licenses/by/4.0/. The Creative Commons Public Domain Dedication waiver (http://creativecommons.org/publicdomain/zero/1.0/) applies to the data made available in this article, unless otherwise stated in a credit line to the data. 
(Continued from previous page)

Conclusion: These findings suggest that male reproductive development may be associated with both essential and non-essential metal exposure during in utero and peripubertal windows.

Keywords: Metal, Hormone, In utero exposure, Pregnancy, Puberty

\section{Introduction}

In recent decades, a trend toward earlier onset of puberty among boys and girls has been described [1-9]. These trends in the timing of puberty, a period of physical and psychological development, have raised concerns regarding the potential impact of environmental factors, including endocrine disrupting chemicals (EDCs) [10-13]. Exposure to EDCs prenatally and at the prepubertal stage are thought to play a role in altered pubertal timing, possibly via their estrogenic or anti-androgenic effects and disruption of normal homeostatic control of the hypothalamicpituitary-gonadal (HPG) or hypothalamic-pituitary-adrenal (HPA) axis [13-26].

Some metals and metalloids, such as cadmium (Cd), lead $(\mathrm{Pb})$, mercury $(\mathrm{Hg})$, and arsenic $(\mathrm{As})$, are non-essential xenobiotics that are known to be harmful to human health [27-30]. These non-essential metal(loid)s are persistent in the environment and children's exposure to them is nearly ubiquitous [31]. Several other metals, such as chromium $(\mathrm{Cr})$, copper $(\mathrm{Cu})$, manganese $(\mathrm{Mn})$, molybdenum $(\mathrm{Mo})$, selenium (Se) and zinc ( $\mathrm{Zn}$ ), are essential for optimal health but may be harmful at insufficient or excessive levels [3236]. A number of metal(loid)s are reproductive toxicants and have endocrine disrupting properties which interfere with many aspects of endocrine functions through interacting with hormone secretion, transport and binding receptors as well as genomic expression and epigenetic modification [19, 37-43]. Some of these proposed mechanisms of actions are common to different metals, such as binding with estrogen receptor $(\mathrm{Cd}, \mathrm{As}, \mathrm{Pb})$ and increasing lipid peroxidation $(\mathrm{Pb}, \mathrm{Hg})$, while others are specific, for instance, stimulation or inhibition of nuclear transcription activity (As) and inhibition of LH secretion (Pb) [44].

Many human and animal studies have focused on elucidating the reproductive effects associated with heavy metal and metalloids, such as $\mathrm{Cd}, \mathrm{Pb}, \mathrm{Hg}$, and As. Though many studies on $\mathrm{Cd}$ were either cross-sectional and/or conducted on adults, there has been some consistency of findings with regard to the positive relationship between male and female $\mathrm{Cd}$ exposure and testosterone concentrations [45-53]. In a group of men with no occupational exposure, positive associations between blood $\mathrm{Pb}$ concentrations and testosterone and/or estradiol levels were reported [46, $48,50]$. In longitudinal studies of children's reproductive development, childhood $\mathrm{Pb}$ exposure was related to later pubertal onset $[54,55]$ and delayed sexual maturation [56] in Russian boys in Chapaevsk, Russia. In contrast, we have shown early life exposure to $\mathrm{Pb}$ was associated with delays in pubertal development in girls but not boys in Mexico City $[21,22]$. Hg was found to be associated with increased estradiol levels in both males and females from a small residential population in Cambodia [57], which was in agreement with a previous study among women with repeated miscarriages [58]. A recent study of As exposure through well water consumption in Taiwan suggested that As may impart an increased risk of erectile dysfunction through a reduction of circulating testosterone [59].

Although less attention has been given to the other metals in the past, a growing body of evidence suggests that certain essential or trace metals, including $\mathrm{Cu}$, fluoride (F), Mn, Mo, and Se can also have adverse effects on male reproduction [60-63]. In addition, most reports on the male reproductive effects of metals are from experimental animal, epidemiological, and occupational studies usually involving high doses not commonly encountered by children. Due to the widespread exposure of humans and known adverse effects related to essential and non-essential metal exposure, concern is growing that low-level exposure may also adversely affect reproductive developmental outcomes in boys. Moreover, only a few studies have investigated the cross-sectional relationships in boys, with the exception of two recent longitudinal studies in Russia and Mexico $[56,63]$, and none have examined exposure during in utero development and subsequent hormone levels during puberty, a time at which steroid hormones play an essential role in reproductive development [11, 64-66].

Therefore, the present study assessed whether in utero and prepubertal exposure to metals at relatively low doses altered reproductive hormone levels or timing and progression of sexual maturation in boys. We extended the limited metals studied on this topic and examined both essential and non-essential metals in relation to reproductive hormone concentrations and progression of sexual maturation in boys from ages $8-14$ years to $10-18$ years.

\section{Method}

\section{Study population}

Participants in this study are part of the "Early Life Exposure in Mexico to Environmental Toxicants (ELEMENT)" project, a longitudinal cohort study of pregnant women in Mexico City and their children [67]. ELEMENT recruited 997 pregnant women from maternity hospitals during their first trimester between 1997 and 2004 and their children, as 
previously described [21, 67-70]. Inclusion criteria included not planning to leave the area within 5 years; no history of infertility, diabetes, or psychosis; not consuming alcoholic beverages daily during pregnancy; no addiction to illegal drugs; no diagnosis of a high-risk pregnancy; being pregnant with singleton. Mothers completed interview-based questionnaires at up to three prenatal visits [mean gestational age at visit 1: 13.5 (range:9-24) weeks, visit 2: 25.1 (range: 19-37) weeks, visit 3: 34.4 (range: 28-43) weeks] and provided spot urine samples at the third trimester visit. Between 2008 and 2011, a subset of their children $(n=$ 250 ), who were then $8-14$ years of age, were contacted to participate in a follow-up study (i.e. early-teen visit). The criteria for eligibility included the availability of archived maternal biological specimens for toxicant assay interest [67]. Between 2013 and 2017, 223 (89\%) of those 250 children participated in a second follow-up study at age 10-18 years (i.e. late-teen visit). Among those 223 children, 109 were boys (92\% retention rate from early to late-teen visit). Figure 1 shows the study design and the timing of biological sample collection and physical examination. Participants provided spot urine and fasting blood samples, anthropometry, and reported socio-demographic information via an interviewer-administered questionnaire. In the current analyses, we included male children who finished the early-teen visit (a majority of these same boys also completed late-teen visit) and had maternal urinary metal concentration measurements and/or their early-teen visit urinary metal measurements available $(n=118)$.

\section{Metal concentrations}

Urinary metal concentrations of 14 metals and metalloids: aluminum (Al), As, barium (Ba), Cd, cobalt (Co), $\mathrm{Cu}$, iron $(\mathrm{Fe}), \mathrm{Mn}, \mathrm{Mo}$, nickel $(\mathrm{Ni}), \mathrm{Pb}$, antimony $(\mathrm{Sb})$, selenium (Se), and $\mathrm{Zn}$ were measured in maternal third trimester urine and urine samples collected during the early-teen visit at age 8-14 years. Prenatal and peripubertal (early-teen) urine samples were collected in sterile cups, aliquoted within 1 hour after collection, frozen and stored at $-80^{\circ} \mathrm{F}$, and shipped on dry ice to McGill University (Montreal, Canada) for analysis. Urinary metals were measured using inductively coupled plasma mass spectrometry (ICPMS; Varian 820-MS, Inc., Palo Alto, California) as described previously [71, 72]. Accuracy and precision were measured using certified reference standards (Institut National de Santé Publique du Québec, or INSPQ) with coefficients of variation (CVs) ranging from 3 to $14 \%$, and each batch run contained procedural blanks and replicate runs [72, 73]. More details regarding quality control (QC) were previously described [73]. Values below the limit of detection (LOD) were replaced with the $\mathrm{LOD} / \sqrt{ } 2$. Urinary specific gravity (SG) was measured using a handheld digital refractometer.

$\mathrm{Pb}$ exposure in this study was also measured in maternal patella/blood and early peripubertal blood, and results of these biomarkers in relation to pubertal development within this population have already been published [21, 22]. As patella and blood $\mathrm{Pb}$ concentrations are better

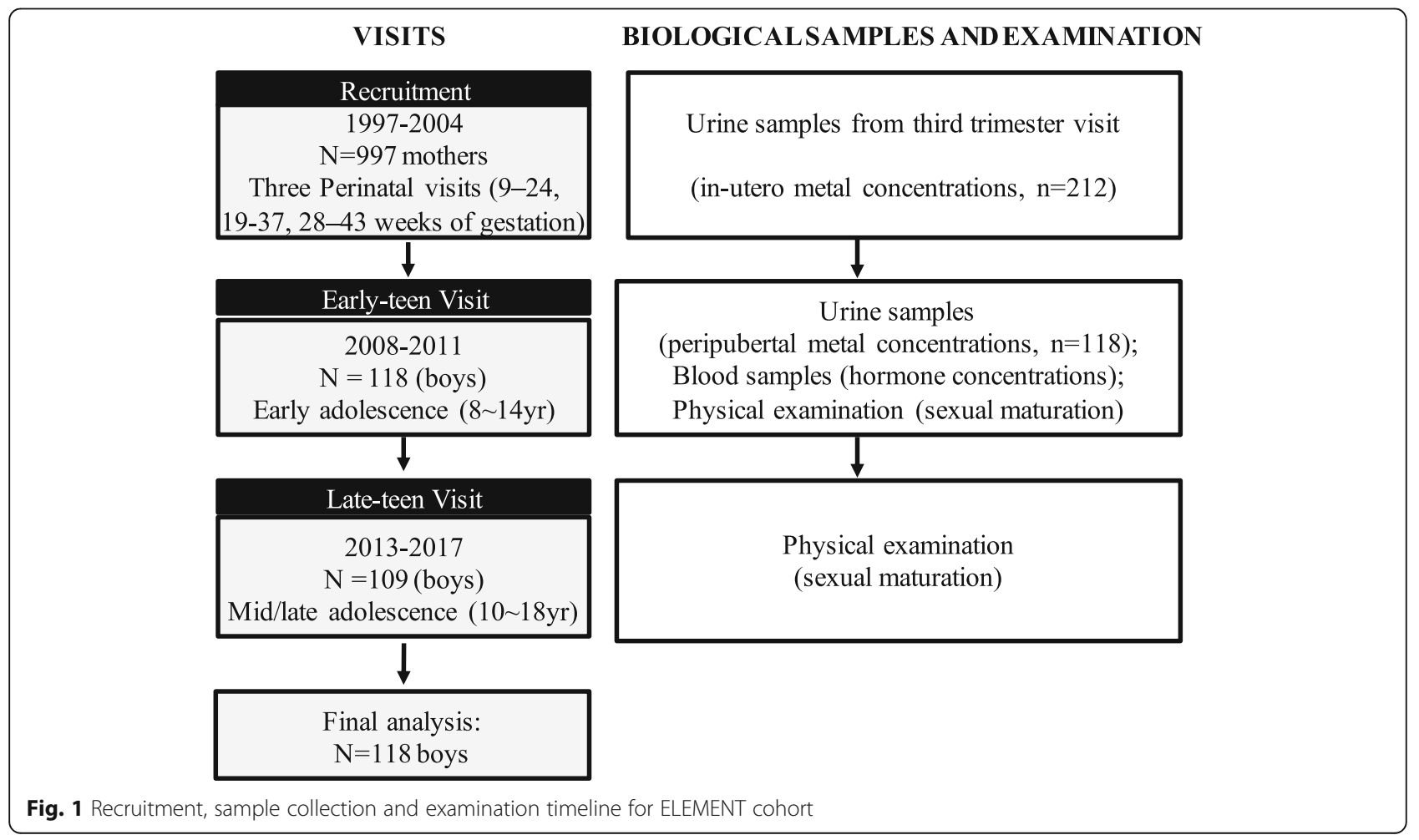


biomarkers of long term $\mathrm{Pb}$ exposure, we excluded urinary $\mathrm{Pb}$ from the current analyses.

\section{Hormones}

Children provided fasting blood samples during the early-teen visit at age 8-14 years. Serum aliquots were separated and frozen at $-80^{\circ} \mathrm{C}$, and then sent to the Clinical Ligand Assay Service Satellite (CLASS) Laboratory at the University of Michigan (Ann Arbor, MI) for hormone analysis. Estradiol, testosterone, inhibin B, and sex hormone-binding globulin (SHBG) were measured in serum samples as biomarkers of puberty, and dehydroepiandrosterone sulfate (DHEA-S) was measured as a biomarker of secretion of adrenal androgens. Estradiol, total testosterone, SHBG, and DHEA-S were measured using an automated chemiluminescent immunoassay (Bayer Diagnostics ACS:180). Active inhibin B was assayed using Gen II ELISA (Beckman Coulter, Webster, TX). All laboratories that performed hormone analyses employed standard quality control (QC) measures, including the use of blanks and duplicate samples to measure instrument precision and identify potential sources of contamination at different collection and measurement stages. The samples were calibrated with standards to determine the degree of bias and implement actions to prevent calibration drift. The laboratories also followed pre-specified protocols for samples that exceed QC activity control limits. Values below the LOD were replaced with the $\mathrm{LOD} / \sqrt{ } 2$.

\section{Sexual maturation}

Two pediatricians evaluated male offspring for Tanner staging and testicular volume using standardized protocols during both follow-up visits at ages 8-14 and 10-18 years. To ensure consistency, pediatricians were trained prior to the start of each follow-up as previously described [74] to evaluate Tanner staging in male participants using standardized protocols. Genital development stage (GD) was assessed as an indicator of puberty and pubic hair stage $(\mathrm{PH})$ as an indicator of adrenarche, with stage 1 corresponding to no development and stage 5 corresponding to full development [75]. Right and left testicular volume (TV) were measured with an orchidometer, and the larger of the two measurements was used in analyses. As a testicular volume of $1-3 \mathrm{~mL}$ is considered prepubertal [76, 77] and $\mathrm{TV} \geq 20 \mathrm{~mL}$ was used as an indicator of sexual maturity [77, 78], cutoffs of $3 \mathrm{~mL}$ and $20 \mathrm{~mL}$ were used to create a 3-level ordinal variable for testicular volume.

\section{Covariates}

Covariates in our analysis included: age at the early-teen visit, BMI z-scores at both visits, and household socioeconomic status at the late-teen visit. BMI z-scores were calculated based on the World Health Organization child reference curves for age and sex (WHO, 2007) for each follow up visit. A 7-level categorical variable for socioeconomic status (SES) was estimated using a validated scale consisting of thirteen questions on housing quality, services, material goods and head of household education (Asociación Mexicana de Agencias de Investigación de Mercados y Opinión Pública, AMAI version $13 \times 6)[79,80]$.

\section{Statistical methods}

The geometric mean concentrations of each metal in urine samples from pregnant women and their children were calculated. The percent of urine samples with concentrations below the LOD were reported for each metal, and metals that were detected in less than $50 \%$ of samples were excluded from further analysis.

Multiple linear regressions were used to assess associations between urinary metal concentrations and peripubertal serum hormone concentrations, where serum hormones were natural log-transformed prior to analysis to achieve normal distribution. Metal concentrations from prenatal and peripubertal urine samples were entered into regression models separately and each model was adjusted for child age and BMI z-score, and for SG as a measure of urinary dilution. Results were calculated as the percent difference in hormone ( $95 \%$ confidence interval) per interquartile range (IQR) increase in urinary metal concentrations. Because reproductive hormones may differ greatly in boys at pre-pubertal stages (Tanner stage $=1)$ vs pubertal stages (Tanner stage $>1$ ), we performed a secondary analysis in which we examined the associations between in utero and peripubertal exposures and reproductive hormone concentrations among subjects who were pre-pubertal (pubic hair Tanner stage $=1, n=94$ ).

Longitudinal analyses were conducted to explore the association between metals and early-teen and late-teen sexual maturation parameters using repeated measures generalized estimating equation (GEE). This allows us to take fuller advantage of the data that have been collected in our longitudinal cohort study and use the additional within-person information to achieve increases in statistical power to detect associations [81]. With separate models for each metal, the GEE approach was used to fit a multinomial (ordinal) regression model for pubertal stages at each visit as a function of metal exposure, age at early-teen visit and change in time, with adjustment for potential confounders:

$$
\begin{aligned}
g\left(E\left[Y_{i j}\right]\right)=\beta_{0}+\beta_{1} M_{i j}+\beta_{2} \text { Age }_{i}+\beta_{3} \text { Time }_{i j} & +\beta_{4} M_{i j} * \text { Time }_{i j} \\
& +\beta_{5} \text { Age }_{i} * \text { Time }_{i j}
\end{aligned}
$$




$$
\begin{aligned}
g\left(E\left[Y_{i j}\right]\right)=\beta_{0} & +\beta_{1} M_{i j}+\beta_{2} \text { Age }_{i}+\beta_{3} \text { Time }_{i j}+\beta_{4} M_{i j} * \text { Time }_{i j} \\
& +\beta_{5} \text { Age }_{i} * \text { Time }_{i j}+\beta_{6} \text { BMIbase }+\beta_{6} \text { BMIvar }
\end{aligned}
$$

Model (1) is the crude model where $Y$ is an outcome of interest (Tanner stages/testicular volume), $g$ is a link function (cumulative logit), $i$ denotes subject number (1, $\ldots, \mathrm{n})$, and $j$ denotes visit number $(1,2) . M$ is $\ln$ transformed metal exposure, Age is the age at the earlyteen visit, Time is the change in time between the earlyteen and late-teen visit. Age at early-teen visit and change in time between two visits were included in the model to account for the effect of baseline age on attained Tanner stage or testicular volume, the natural pubertal progression across time, and the effect of baseline age on the natural pubertal progression. Model (2) is the final adjusted model, in which BMI $\mathrm{z}$-score at the early-teen visit (BMIbase) and the change in BMI $\mathrm{z}$ score from early-teen to late-teen visit (BMIvar) were included. The coefficients of interest are the crosssectional effect of metal on tanner stage/testicular volume $\left(\beta_{1}\right)$ and the effect of metal on the progression of tanner stage/testicular volume over time $\left(\beta_{4}\right)$. Given the possibility that age and BMI z-score at the early-teen visit may be associated with Tanner stage and influence the future progression of Tanner stage, working independence was chosen as the covariance structure to ensure the validity of parameter estimates [82]. We ran the GEE models both with and without BMI z-score (model 1 and 2) as BMI may be on the causal pathway between exposure and puberty. However, the magnitude of estimates from both models were almost identical, therefore we reported results from the models including BMI zscore. Results are presented as odds ratios (OR) and 95\% confidence intervals (95\% CI) per IQR increase in exposure. As few participants were categorized as having a $\mathrm{PH}$ Tanner stage $=3$ and stage $=4$ during the early-teen visit, the number of covariates we could reliably enter into models was limited [83, 84]. Thus, to minimize the number of covariates in GEE models, we included SGcorrected metal concentrations (rather than entering SG as a separate covariate which yielded similar results), using the following equation: $\mathrm{P}_{\mathrm{c}}=\mathrm{P}\left[\left(\mathrm{SG}_{\mathrm{p}}-1\right) /\left(\mathrm{SG}_{\mathrm{i}}-1\right)\right]$ where $P_{c}$ is the $S G$ corrected metal concentration $(\mu \mathrm{g} /$ $\mathrm{L}), P$ is the measured metal concentration, $S_{\mathrm{p}}$ is the median urinary specific gravity, and $\mathrm{SG}_{\mathrm{i}}$ is the individual's urinary specific gravity.

Because SES could be a potential confounder, we ran a sensitivity analysis including SES as a covariate in models of hormones and maturation stages. Furthermore, as both low and high levels of essential metals are of concern, to explore potential non-linear associations we used adjusted generalized additive models (GAM) to graphically depict the cross-sectional relationship between metal concentrations and hormones and sexual maturation measurements. We also considered significance after adjusting for multiple testing using the Benjamini-Hochberg method [85]. Since Tanner stages/ testicular volumes were not independent of each other, we calculated $q$ values (adjusted $p$ values) treating each outcome as a family of tests (11 tests per outcome). A cutoff of 0.15 for $q$ value was used to further interpret main results with greater confidence. All analyses were performed using $R$ version 3.5.2 and SAS 9.4.

\section{Results}

\section{Demographics and exposure distributions}

Characteristics of participants in the original cohort of 997 women were previously described in detail [67]. Mothers included in our analysis had similar demographic characteristics to the overall ELEMENT population; the mean age of mothers at the time of enrollment was 26.6 (standard deviation $=5.3$ ). Mothers had on average 11 years of education, most were married or cohabitating (89\%), and all lived within Mexico City. Very few (3\%) reported smoking during pregnancy. Average age of the boys at the early-teen and late-teen visits were 10.4 and 13.7, respectively. Mean and standard deviation of the follow up period were 3.5 and 0.5 years. The percent of samples with metal concentrations below the limit of detection, as well as the geometric means, standard deviations, and selected percentiles of sample concentrations from prenatal and peripubertal (early-teen) visits are shown in Table 1. Spearman correlations between the prenatal and peripubertal visit metal concentrations adjusted for SG are also presented in the table. With the exception of $\mathrm{Sb}$ and $\mathrm{Fe}$, other metals were detected in $>50 \%$ of urine samples, therefore, $\mathrm{Sb}$ and $\mathrm{Fe}$ were excluded from further analysis. Weak correlations between maternal and peripubertal metal concentrations and weak to moderate correlations between different urinary metal concentrations within maternal and child samples have been previously reported elsewhere [73].

\section{Hormone and Tanner stages distributions}

Distributions of reproductive hormones among ELEM ENT boys were described previously [86]. Except for 12 total testosterone measurements, all measures were above the LOD. Spearman correlations among hormones were weak to moderate ( $R=-0.42$ to 0.63$)$. Distributions of Tanner stages of sexual maturation and testicular volume among the male children at the two follow up visits are reported (Supplementary Table S1). We additionally provided spaghetti plots depicting Tanner stage and testicular volume progression between two visits (Supplementary Fig. S1), and the distribution of measures of sexual maturation for different age groups of ELEMENT 
Table 1 Distribution of urinary metal concentrations $(\mu \mathrm{g} / L)$ among ELEMENT mothers and their male children at age 8-14 years ${ }^{\mathrm{a}}$

\begin{tabular}{|c|c|c|c|c|c|c|c|c|c|c|c|c|c|c|c|c|}
\hline \multirow{2}{*}{ 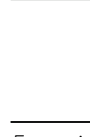 } & \multicolumn{8}{|c|}{ In utero } & \multicolumn{7}{|c|}{ Peripubertal } & \multirow[b]{2}{*}{$P$ value } \\
\hline & LOD & $\begin{array}{l}\%< \\
\text { LOD }\end{array}$ & GM & GSD & $25 \%$ & $50 \%$ & $75 \%$ & $\operatorname{MAX}$ & $\%<$ LOD & GM & GSD & $25 \%$ & $50 \%$ & $75 \%$ & MAX & \\
\hline \multicolumn{17}{|c|}{ Essential metals } \\
\hline Co & 0.4 & 0.0 & 1.2 & 1.9 & 0.8 & 1.2 & 2.1 & 5.2 & 0.0 & 0.7 & 1.5 & 0.6 & 0.8 & 0.9 & 1.6 & 0.63 \\
\hline $\mathrm{Cu}$ & 48.2 & 46.3 & 86.1 & 4.4 & 34.1 & 50.2 & 71.9 & 2742 & 56.8 & 43.3 & 1.3 & 34.1 & 34.1 & 56.1 & 106 & 0.83 \\
\hline $\mathrm{Mn}$ & 0.4 & 7.4 & 0.8 & 1.7 & 0.6 & 0.7 & 1.0 & 8.7 & 2.5 & 1.2 & 1.8 & 0.8 & 1.2 & 1.7 & 4.4 & 0.68 \\
\hline Mo & 2.9 & 15.8 & 19.5 & 3.3 & 12.7 & 25.7 & 42.9 & 308 & 0.0 & 46.6 & 1.8 & 33.7 & 50.2 & 67.1 & 210 & 0.99 \\
\hline $\mathrm{Se}$ & 10.5 & 3.2 & 29.9 & 1.7 & 23.0 & 32.6 & 43.1 & 120 & 1.7 & 47.2 & 1.7 & 36.3 & 53.0 & 65.6 & 141 & 0.26 \\
\hline $\mathrm{Zn}$ & 0.1 & 1.2 & 271 & 2.2 & 160 & 298 & 451 & 1253 & 0.0 & 366 & 1.8 & 257 & 411 & 521 & 1200 & 0.08 \\
\hline \multicolumn{17}{|c|}{ Non-essential Metals } \\
\hline Al & 8.6 & 11.6 & 24.0 & 2.5 & 12.3 & 20.3 & 42.2 & 304 & 24.6 & 14.5 & 2.1 & 8.8 & 14.0 & 23.3 & 428 & 0.37 \\
\hline As & 0.3 & 0.0 & 14.0 & 2.0 & 9.3 & 13.2 & 20.6 & 153 & 0.0 & 14.3 & 2.0 & 10.2 & 14.4 & 20.5 & 515 & 0.07 \\
\hline $\mathrm{Ba}$ & 1.1 & 3.2 & 4.0 & 2.0 & 2.6 & 4.2 & 5.9 & 27.6 & 10.2 & 2.5 & 2.0 & 1.6 & 2.4 & 3.9 & 20.2 & 0.81 \\
\hline $\mathrm{Ni}$ & 3.0 & 0.0 & 8.7 & 1.9 & 5.8 & 7.4 & 11.0 & 107 & 0.8 & 8.1 & 1.6 & 5.9 & 8.0 & 10.8 & 53.2 & 0.83 \\
\hline $\mathrm{Cd}$ & 54.0 & 2.1 & 0.2 & 2.1 & 0.1 & 0.2 & 0.3 & 2.7 & 2.5 & 0.1 & 1.6 & 0.1 & 0.1 & 0.2 & 0.3 & 0.24 \\
\hline
\end{tabular}

${ }^{a}$ uncorrected for specific gravity

${ }^{\text {b }} P$ value from Spearman correlation test between in utero and peripubertal metal concentration measurements

boys (Supplementary Table S2). At the early -teen visit, the majority of the boys $(n=94,81.7 \%)$ were at Tanner stage 1 for pubic hair development whereas 57 boys (49.6\%) were at Tanner stage 1 for genital development. Most boys who were at Tanner stage 1 moved to more advanced Tanner stages at the late-teen visit- only 28 (26.4\%) and $8(7.5 \%)$ were still at Tanner stage 1 for pubic hair development and genital development after 3 years on average since the early-teen visits. Boys who were at Tanner stages 2,3 , and 4 at the early-teen visit all progressed to higher stages at the late-teen visit, with $14(13.2 \%)$ and $18(13.2 \%)$ boys reaching full development $($ Tanner stage $=5)$ for the two measurements. In terms of testicular volume distribution, the percentage of the boys in the prepubertal stage dropped from 14.8 to $0 \%$ from early-teen to late-teen visit.

\section{In utero and peripubertal metal exposure and peripubertal hormone concentrations}

Associations between in utero and peripubertal metal concentrations and reproductive hormones are presented in Fig. 2 and Supplementary Table S4. Positive associations were observed between some urinary essential metal concentrations and estradiol, testosterone, and SHBG. One IQR increase in in utero $\mathrm{Zn}$ concentration was associated with $13.7 \%$ higher serum estradiol (95\% CI: $0.3,28.8$ ). In utero Co and Mn were positively associated with SHBG concentrations, with an IQR increase associated with $16 \%$ (95\% CI:0.4, 34.2) and 14.2\% (95\%CI: 1.5, 28.5) higher serum SHBG after adjustment for child age, BMI z-score, and SG, respectively. As shown in Fig. 2, effect estimates for the association between both in utero essential and non-essential metal concentrations and testosterone were larger compared to other reproductive hormones, $51.3 \%$ for Mo, $35 \%$ for As, $38.9 \%$ for $\mathrm{Cd}$. In models where reproductive hormones were regressed on concurrent peripubertal exposures, the strongest associations were observed also between metal concentrations and testosterone, particularly with non-essential metals $\mathrm{Ni}(\% \Delta / \mathrm{IQR}$ : $35.1,95 \%$ CI: $2.6,77.8)$ and $\mathrm{Ba}(\% \Delta / \mathrm{IQR}: 59.1,95 \%$ CI: $22.5,106.8)$. Peripubertal $\mathrm{Ba}$ concentration was also associated with higher estradiol (\% $\% / \mathrm{IQR}: 10.2,95 \%$ CI: $0.5,20.9)$. However, no significant associations were detected between peripubertal metal exposures and DHEA-S or inhibin B.

After correcting for multiple testing, the associations of in utero Mo, As, and $\mathrm{Cd}$ with testosterone, as well as the association of peripubertal $\mathrm{Ba}$ with testosterone had qvalues $<0.15$ (Fig. 2 and Supplementary Table S4), providing greater confidence in these associations.

\section{In utero and peripubertal metal exposure and sexual maturation}

We have presented results from multiple ordinal regression models of in utero metal concentrations and Tanner stage and testicular volume in Fig. 3 and Supplementary Table S5. Tanner stages and testicular volume at the early-teen visit were not associated with in utero metal concentrations, with the exception of a nonessential metal, $\mathrm{Al}$; an IQR increase in in utero $\mathrm{Al}$ concentrations was associated with 3.6 times greater odds ( $95 \%$ CI: $1.67,7.76)$ of being at a higher category of testicular volume versus lower categories. In the same figure and table, associations between in utero metal 


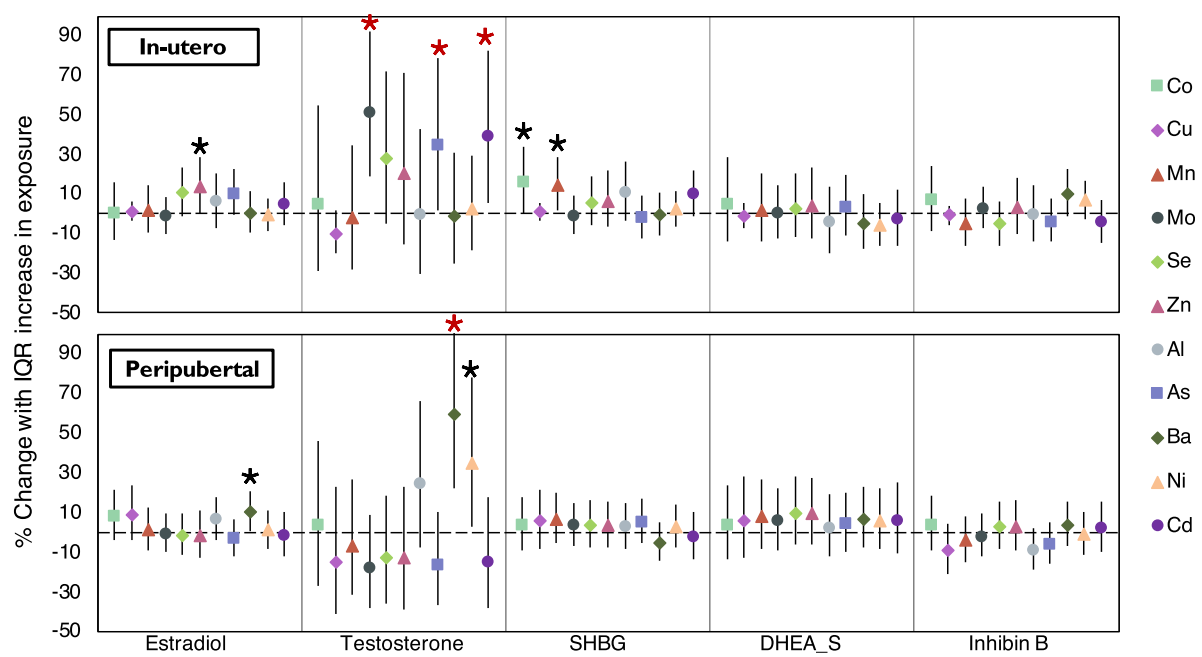

Fig. 2 Peripubertal hormone concentrations associated with in utero and peripubertal metal concentration among ELEMENT boys ${ }^{\mathrm{ab}}$. ${ }^{\mathrm{a}}$ Results are presented as the percent difference in peripubertal hormone concentrations associated with an interquartile range (IQR) increase in in utero and peripubertal metal. ${ }^{\mathbf{b}}$ Linear regression models were adjusted for child age, BMI $z$-score and specific gravity. $*$ Significant associations with $p$ value $<0.05 \& q$ value $<0.15$. Significant associations with $p$ value $<0.05 \& q$ value $>0.15$

concentrations and pubertal development over time in boys are also presented. During the follow-up, an IQR increase in in utero concentrations of non-essential metalloid As was associated with 36\% (OR/IQR: 0.64, 95\% CI: 0.48, 0.85) lower odds of genital development progression per year, adjusting for age, BMI, and Tanner stage at the early-teen visit (GEE longitudinal model). In utero concentrations of non-essential metal (loid) s Al (OR/IQR: 0.61, 95\% CI: 0.45, 0.83) and As (OR/IQR:
0.64, 95\% CI: 0.43, 0.97) were associated with lower odds of progressing to a higher testicular volume category (i.e. 39 and 36\% lower odds/IQR). Essential metal Zn was also associated with $38 \%$ lower odds of testicular volume progression (OR/IQR: 0.62, 95\% CI: 0.44, 0.88).

Similarly, Fig. 4 and Supplementary Table S5 show the cross-sectional and longitudinal associations between prepubertal metal concentrations and sexual maturation and progression. No significant associations were found

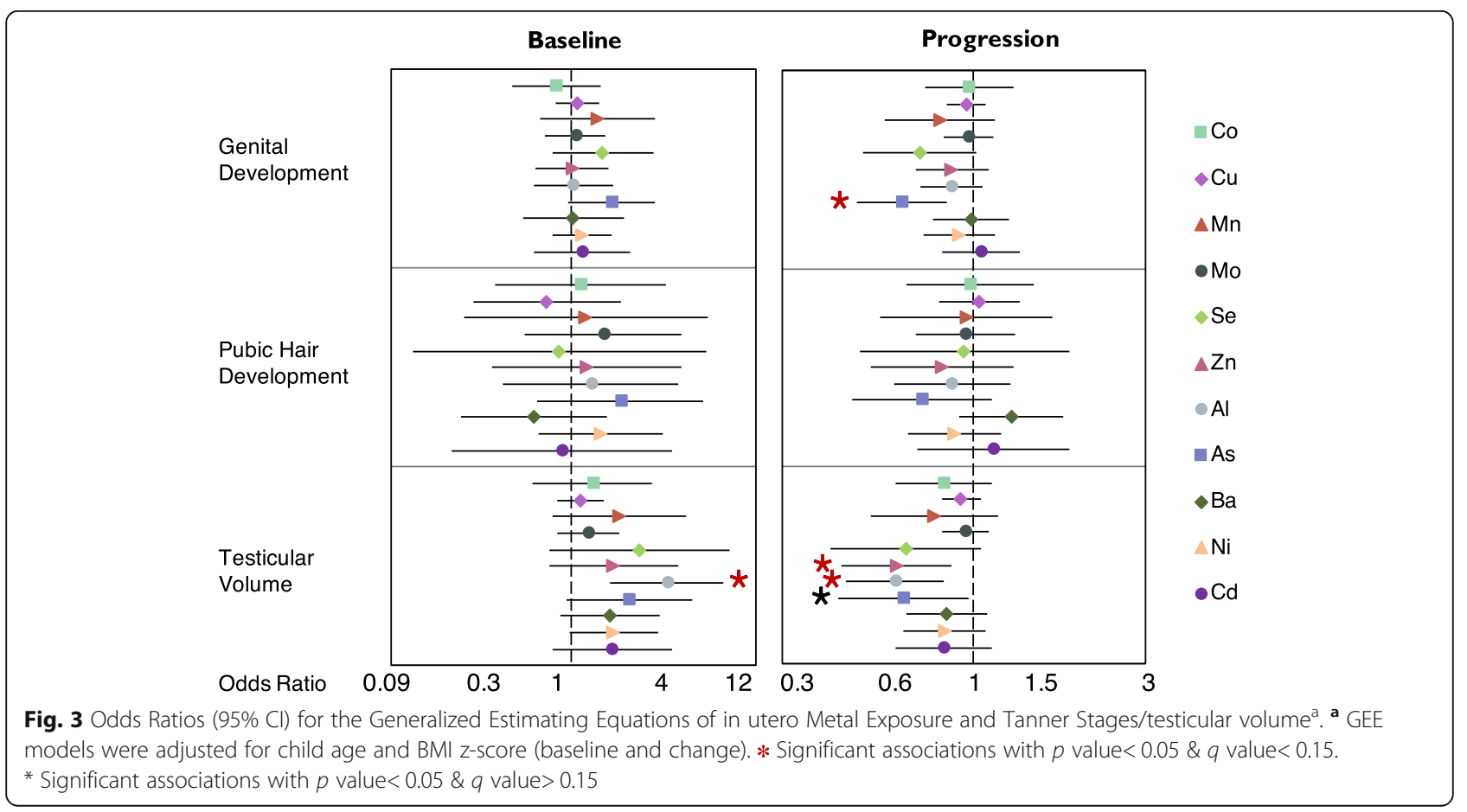




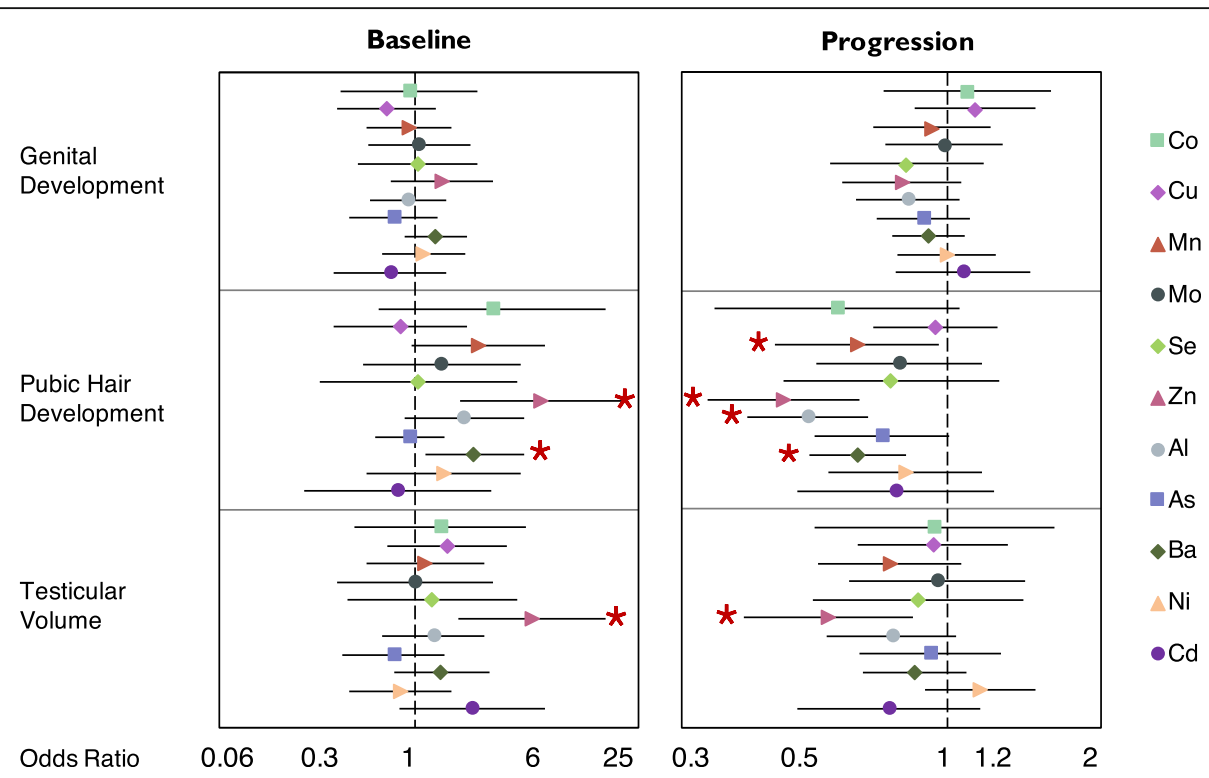

Fig. 4 Odds Ratios $\left(95 \% \mathrm{Cl}\right.$ ) for the Generalized Estimating Equations of peripubertal Metal Exposure and Tanner Stages/testicular volume ${ }^{\mathrm{a}}$. ${ }^{\mathrm{a}} \mathrm{GEE}$ models are adjusted for child age and BMI z-score (baseline and change). $*$ Significant associations with $p$ value $<0.05 \& q$ value $<0.15$.

* Significant associations with $p$ value $<0.05 \& q$ value $>0.15$

between peripubertal urinary metal concentrations with Tanner stage for genital development or progression over follow-up, although several essential and nonessential metals were associated with pubic hair Tanner stages, testicular volume, and progressions. Peripubertal $\mathrm{Zn}$ concentration was associated with higher odds of being at a higher developmental stage for pubic hair (OR/IQR: 6.11, 95\% CI: 1.89, 19.69) and testicular volume (OR/IQR: 5.39, 95\% CI: 1.88, 15.49) at the early-teen visit, as well as slower progression of pubic hair development (OR/IQR: 0.47, 95\% CI: 0.34, 0.67) and testicular volume (OR/IQR: 0.58, 95\% CI: 0.40, $0.85)$ during the follow-up. Higher Mn was associated with 34\% (OR/IQR: 0.66, 95\% CI: 0.46, 0.96) lower pubic hair development progression only. Regarding non-essential metals, an IQR increase in peripubertal $\mathrm{Ba}$ concentration was associated with 2.3 times greater odds (95\% CI: 1.15, 4.75) of being at higher Tanner stage for pubic hair development at age 8-14 years, but with 34\% (OR/IQR: $0.66,95 \%$ CI: 0.53 , 0.83 ) lower odds of progressing to a higher pubic hair Tanner stage per year of follow-up. Higher peripubertal urinary $\mathrm{Al}$ was also associated with $47 \%$ (OR/IQR: 0.53, 95\% CI: $0.40,0.70$ ) lower odds of pubic hair development progression per IQR increase.

All the associations described above had $q$-values < 0.15 (Fig. 3, Fig. 4, and Supplementary Table S5) after correcting for multiple testing, except for the association between in utero As and testicular volume progressions. Output graphics produced from GAM models showed that there was no significant non-linear relationship between essential and non-essential metal concentrations and hormones and sexual maturation outcomes, after adjusting for the same sets of covariates.

\section{Sensitivity analysis}

Results from the hormone subset analysis when we restricted to boys who were prepubertal (94 out of 118) at the early-teen visit are similar to the main analysis results (Supplementary Table S6). For models regressing in utero metal concentrations, some effect estimates for DHEA_S, SHBG, and testosterone were no longer significant, potentially due to the small sample size. For models regressing peripubertal exposure, the notable effect estimates for testosterone were similar to those from the main analysis. The association between DHEA_S and $\mathrm{Ni}(\% \Delta / \mathrm{IQR}=20.2$, $95 \% \mathrm{CI}=1.7,42.0)$ and inhibin $\mathrm{B}$ and $\mathrm{Al}(\% \Delta / \mathrm{IQR}=-19.2$, $95 \% \mathrm{CI}=-31.7,-4.4)$ became stronger and significant in the peripubertal subset analysis.

The magnitude of estimates from GEE models with and without BMI (Supplementary Table S7) were almost identical. Findings from models adjusting for SES were generally consistent with metal and hormone associations observed in our main analyses; in SES-adjusted models, the association between in utero Co concentrations and higher SHBG $(\% \Delta / \mathrm{IQR}=14.2,95 \% \mathrm{CI}=-2.9,23.3)$ was slightly attenuated and no longer significant, while the association between peripubertal $\mathrm{Al}$ and inhibin $\mathrm{B}$ $(\% \Delta / \mathrm{IQR}=-14.1,95 \% \mathrm{CI}=-24.6,-2.2)$ was stronger and significant. In GEE models for Tanner stage or testicular volume status including SES finding remain consistent with the main models. 
A high proportion of samples had metal concentrations above the LOD except for $\mathrm{Cu}$, which was below the detection limit in $46 \%$ of samples. In a secondary analysis, we categorized urinary $\mathrm{Cu}$ concentrations into three groups. The low group consisted of values below the LOD, while the medium and high groups were made up of equalized bins among the detected values. We estimated the model parameters again and found that they were similar to the main parameter estimates.

\section{Discussion}

Previous studies of metal exposure and male reproductive development have primarily been cross-sectional and focused on heavy metals, with the exception of "the Russian Children's Study" and "Early Life Exposure in Mexico to Environmental Toxicants (ELEMENT) Study" that assessed the longitudinal impact of blood $\mathrm{Pb}[56$, 63]. Thus, it is critical to evaluate the associations of essential and non-essential metals with reproductive hormones, sexual maturation, and progression among boys. Based on our longitudinal analysis of boys in a birth cohort in Mexico City who were examined at two peripubertal follow-up visits, we demonstrated a higher pubic hair development stage and testicular volume at the early-teen visit with higher non-essential metal concentrations, including in utero $\mathrm{Al}$ and peripubertal $\mathrm{Ba}$ and essential metal $\mathrm{Zn}$ concentration (peripubertal). We also found associations between in utero and peripubertal exposure to a number of both essential and non-essential metals and slowed progression of pubertal development across the follow-up period. In relation to hormone concentrations, higher non-essential metals, in utero As and $\mathrm{Cd}$ and peripubertal Ba concentrations as well as essential metal Mo measured in utero were associated with substantially higher testosterone. None of the associations between metals and hormones and measurements of sexual maturations appear to be non-linear.

To our knowledge, this is the first study to investigate the association between various in utero and peripubertal metals measured in urine and reproductive hormone concentrations in teenage boys. We reported a number of positive associations between specific metals measured in the urine and reproductive hormones, particularly testosterone. Some of our findings have not been reported elsewhere, such as relationships of essential minerals, e.g., $\mathrm{Zn}$ with estradiol or Co and Mn with SHBG. The positive association between relatively low $\mathrm{Cd}$ exposure and testosterone in this study is supported by epidemiological studies of non-occupational exposures among men [46, 48-50]. However, in vivo and in vitro observations linked $\mathrm{Cd}$ with altered adrenal gland activity and induced oxidative stress, which could result in toxic effects on the testis and subsequently decrease in serum testosterone [87-91].
Mo is an essential nutrient that is a cofactor for important enzymes involving in toxin removal [92, 93]. While no prior studies have investigated the associations between Mo and hormones in boys, contrary to our finding on in utero Mo and increased testosterone, decreased testosterone and sperm concentrations were observed in males with increasing levels of Mo [50, 60, 94]. Several animal studies have also reported the reproductive toxicity of Mo, including declines in sperm concentration, motility and normal morphology, testicular degeneration, and reduced fertility [95-98]. It is worth noting that the prenatal Mo concentrations were lower in our study population compared to the previous studies of Mo and reproductive outcomes and those reported in the National Health and Nutrition Examination Survey (NHANES) [99] (Supplementary Table S3). It is not clear whether Mo concentrations measured in utero will have different effects on reproductive hormones measured during different life stages, which warrants further investigation. Although previous studies have not evaluated associations between $\mathrm{Ba}$ and reproductive hormones in human, our finding of positive associations between peripubertal $\mathrm{Ba}$ concentrations and serum testosterone and estradiol is consistent with previous studies among male zebrafish, where exposure to barium chloride significantly increased estradiol concentrations and transcripts of genes involved in the HPG axis [100].

One of the main observations in this study is that $\mathrm{Zn}$ is associated with higher odds of pubic hair and testicular volume stage at the early-teen follow-up, but a slower progression of puberty from the early-teen to late-teen visit. Few cross-sectional studies on boys and girls also reported a significant positive relationship between serum or plasma zinc concentrations and stages of sexual maturation [101-104], as well as reproductive hormones (testosterone). $\mathrm{Zn}$ is an essential trace metal and is fundamental for the development of the male reproductive system $[105$, 106]. Studies on various animals described the role of $\mathrm{Zn}$ in advancing male puberty, through increased testicular activity, testosterone production, metabolism, and growth, primarily via activated the hypothalamus and the pituitary functions [107-112]. However, we did not observe a significant association between serum $\mathrm{Zn}$ and testosterone concentrations.

Pubic hair stages at the early-teen visit and progression were consistently associated with elevated concentrations of certain peripubertal metals, including $\mathrm{As}, \mathrm{Al}, \mathrm{Mn}$, and $\mathrm{Zn}$. The biological mechanisms that underlie the link between these essential and non-essential metals and pubertal development are uncertain but may be related to reproductive hormones. The main hormones responsible for pubic hair development in males are the androgens DHEA and testosterone [113, 114]. In this cohort, the strongest observed associations were between in utero and 
peripubertal metal concentrations and increased testosterone, while no significant associations were seen in relation to DHEA-S. It is possible that these metals impact the appearance of pubic hair through changes in testosterone.

In this study, we observed slower progression of sexual maturation for those boys at higher Tanner stages at the early-teen visit which was also reported in studies explored the association between pubertal onset and progression [115-117]. A compensatory mechanism similar to "catch-up growth" was proposed previously as a potential explanation for this observation [68, 70]. For those boys who had experienced a delayed pubertal development, their body systems may have responded by accelerating the tempo of pubertal progression (the change from lower stages of puberty to higher stages); while others who had experienced an advanced pubertal development may respond by slowing down the pubertal progression. This concept may explain the associations between metal exposure, earlier puberty onset at earlyteen, and slower progression we found in this study. Future research is needed to establish this phenomenon and potential mechanisms.

Several of the associations with hormones and sexual maturation presented in this study are comparable to previous reports from human and animal research and supported by the current understanding of male pubertal development. However, there are inconsistent findings between the current study and previous studies and this may due to a number of reasons; 1 ) the exposure assessment approaches were different [sampling period, exposure matrices (i.e., blood, urine, hair)]. 2) most of the previous studies exploring the relationship between metals and hormones were conducted on adult population. 3) most studies examined the cross-sectional relationships whereas we examined the longitudinal association between metals and sexual maturation and progression. It is also worth noting that the assessment of sexual maturation markers in this study was conducted by the same observers at both visits in this study to minimize measurement bias. Limitations of our study include a somewhat small sample size and few observations for certain Tanner stages, which may result in imprecise effect estimates. The age range of children at the early-teen and late-teen visits does overlap, however, our results are unlikely biased as the average follow up period for different age groups is 3.5 years. Some of the metals measured in this study have a relatively short half-life in urine, so urinary concentrations at the time measurement may not fully characterize exposure during each specific window of development. Lastly, hormone concentrations were only measured at one time point and are likely to be subject to nondifferential misclassification due to diurnal variation. Further research is warranted to prospectively explore the underlying mechanisms by which metals may affect male sexual maturation and progression in larger study populations.

\section{Conclusions}

The ELEMENT study, a prospective longitudinal birth cohort study in Mexico City, provided an opportunity to study the relationships of both in utero and peripubertal metal exposure on hormone concentrations and measures of sexual maturation and progression during the peripubertal period. Our results indicate that the in utero and peripubertal periods are vulnerable life stages, during which metal exposures may lead to disruption of male reproductive hormones and pubertal development. The findings also support that essential and nonessential metals have the potential to disrupt the onset and progression of puberty via interrupting the critical hormonal pathways.

\section{Supplementary Information}

The online version contains supplementary material available at https://doi. org/10.1186/s12940-020-00672-0.

\section{Additional file 1.}

\section{Acknowledgments}

The authors acknowledge the research staff at participating hospitals and the American British Cowdray Hospital in Mexico City for providing research facilities. We thank the mothers and children for participating in the study.

\section{Authors' contributions}

PA conducted data analyses and drafted the manuscript. JDM, KEP, MTR and DJW conceived the study and oversaw its coordination. NB helped oversee the study, data acquisition, metals analysis, and advised the interpretation and drafting of the manuscript. BNS helped oversee the study and advised during the data analysis. MSG and AMG participated in the collection and assembly of data. The authors read and approved the final manuscript.

\section{Funding}

This work was supported by U.S. Environmental Protection Agency (US EPA) grants RD834800 and RD83543601 and National Institute for Environmental Health Sciences (NIEHS) grants P20 ES018171, P01 ES02284401, and P30 ES017885. Its contents are solely the responsibility of the grantee and do not necessarily represent the official views of the US EPA. Further, the US EPA does not endorse the purchase of any commercial products or services mentioned in the publication. This work was also supported and partially funded by the National Institute of Public Health, Ministry of Health of Mexico.

\section{Availability of data and materials}

All data generated and analyzed during this study are not publicly available due to the Institutional Review Board restrictions.

\section{Ethics approval and consent to participate}

Research protocols were approved by the Institutional Review Board at University of Michigan, and the Research, Biosafety and Ethics in Research at the Mexico National Institute of Public Health. Prior to enrollment, informed consent from mothers and informed assent from offspring were obtained.

Consent for publication

Not applicable.

Competing interests

The authors declare that they have no competing interests. 


\section{Author details}

'Department of Environmental Health Sciences, University of Michigan School of Public Health, 1415 Washington Heights, Ann Arbor, Ml 48109, USA. ${ }^{2}$ Department of Biostatistics, University of Michigan School of Public Health, Ann Arbor, MI, USA. ${ }^{3}$ Faculty of Agricultural and Environmental Sciences, McGill University, Montreal, Quebec, Canada. ${ }^{4}$ Center for Nutrition and Health Research, Instituto Nacional de Salud Pública, Cuernavaca, Morelos, Mexico. ${ }^{5}$ Mexican Council for Science and Technology, Mexico City, Mexico. ${ }^{6}$ Department of Nutritional Sciences, University of Michigan School of Public Health, Ann Arbor, MI, USA.

\section{Received: 10 June 2020 Accepted: 30 October 2020} Published online: 25 November 2020

\section{References}

1. Anderson SE, Dallal GE, Must A. Relative weight and race influence average age at menarche: results from two nationally representative surveys of US girls studied 25 years apart. Pediatrics. 2003;111(4 Pt 1):844-50.

2. Herman-Giddens ME, et al. Secondary sexual characteristics and menses in young girls seen in office practice: a study from the pediatric research in office settings network. Pediatrics. 1997;99(4):505-12.

3. Wyshak G, Frisch RE. Evidence for a secular trend in age of menarche. $N$ Engl J Med. 1982;306(17):1033-5.

4. Euling SY, et al. Examination of US puberty-timing data from 1940 to 1994 for secular trends: panel findings. Pediatrics. 2008;121(Suppl 3):S172-91.

5. Karpati AM, et al. Stature and pubertal stage assessment in American boys: the 1988-1994 third National Health and nutrition examination survey. J Adolesc Health. 2002;30(3):205-12.

6. Herman-Giddens ME, Wang L, Koch G. Secondary sexual characteristics in boys: estimates from the national health and nutrition examination survey III, 1988-1994. Arch Pediatr Adolesc Med. 2001;155(9):1022-8.

7. Wacharasindhu S. A trend of normal puberty around the world. Siriraj Med J. 2017:61(1):1-2

8. Paris F, Gaspari L, Sultan C. Precocious Puberty and Environmental Endocrine Disruptors. In: Bouvattier C, Pienkowski C, editors. Early Puberty. Paris: Springer; 2016. https://doi.org/10.1007/978-2-8178-0543-6_2.

9. Lee EJ, et al. A study on the recent trend of chief complaint of Korean pediatric and adolescent outpatients. J Pediatr Kor Med. 2016;30(1):45-58.

10. Blakemore SJ, Burnett S, Dahl RE. The role of puberty in the developing adolescent brain. Hum Brain Mapp. 2010;31(6):926-33.

11. Sisk CL, Foster DL. The neural basis of puberty and adolescence. Nat Neurosci. 2004;7(10):1040-7.

12. Lee $Y$, Styne D. Influences on the onset and tempo of puberty in human beings and implications for adolescent psychological development. Horm Behav. 2013;64(2):250-61.

13. Buck Louis GM, et al. Environmental factors and puberty timing: expert panel research needs. Pediatrics. 2008;121(Suppl 3):S192-207.

14. Bellingham $\mathrm{M}$, et al. Exposure to a complex cocktail of environmental endocrine-disrupting compounds disturbs the kisspeptin/GPR54 system in ovine hypothalamus and pituitary gland. Environ Health Perspect. 2009; 117(10):1556-62

15. Jacobson-Dickman E, Lee MM. The influence of endocrine disruptors on pubertal timing. Curr Opin Endocrinol Diabetes Obes. 2009;16(1):25-30.

16. Den Hond E, Schoeters G. Endocrine disrupters and human puberty. Int J Androl. 2006;29(1):264-71 discussion 286-90.

17. Roy JR, Chakraborty S, Chakraborty TR. Estrogen-like endocrine disrupting chemicals affecting puberty in humans--a review. Med Sci Monit. 2009;15(6): RA137-45.

18. Massart F, et al. How do environmental estrogen disruptors induce precocious puberty? Minerva Pediatr. 2006;58(3):247-54.

19. Diamanti-Kandarakis $\mathrm{E}$, et al. Endocrine-disrupting chemicals: an Endocrine Society scientific statement. Endocr Rev. 2009;30(4):293-342.

20. Pescovitz $\mathrm{OH}$, Walvoord EC, eds. When puberty is precocious: scientific and clinical aspects. Springer Science \& Business Media; 2007

21. Liu Y, et al. Early lead exposure and pubertal development in a Mexico City population. Environ Int. 2019;125:445-51.

22. Jansen EC, et al. Prenatal lead exposure in relation to age at menarche: results from a longitudinal study in Mexico City. J Dev Orig Health Dis. 2018;9(4):467-72.

23. Parent AS, Franssen D, Fudvoye J, Pinson A, Bourguignon JP. Current Changes in Pubertal Timing: Revised Vision in Relation with Environmental
Factors Including Endocrine Disruptors. Endocrine Development. 2016;29: 174-84. https://doi.org/10.1159/000438885.

24. Fudvoye J, Lopez-Rodriguez D, Franssen D, Parent A-S. "Endocrine disrupters and possible contribution to pubertal changes." Best Pract Res Clin Endocrinol Metab. 2019;33(3):101300.

25. Watkins DJ, et al. Phthalate and bisphenol a exposure during in utero windows of susceptibility in relation to reproductive hormones and pubertal development in girls. Environ Res. 2017;159:143-51.

26. Watkins DJ, et al. Relating phthalate and BPA exposure to metabolism in peripubescence: the role of exposure timing, sex, and puberty. J Clin Endocrinol. 2016;101(1):79-88.

27. Agency for Toxic Substances and Disease Registry (ATSDR). Toxicological profile for Cadmium. Atlanta, GA: U.S. Department of Health and Human Services, Public Health Service. 2012.

28. Agency for Toxic Substances and Disease Registry (ATSDR). Toxicological profile for Arsenic. Atlanta, GA: U.S. Department of Health and Human Services, Public Health Service. 2007.

29. Atsdr U. Toxicological profile for lead (Atlanta, GA: US Department of Health and Human Services, Agency for Toxic Substances and Disease Registry (ATSD R), public health service)US EPA (2006) Air quality criteria for lead; 2007.

30. $\mathrm{Ha} \mathrm{E}$, et al. Current progress on understanding the impact of mercury on human health. Environ Res. 2017;152:419-33.

31. Claus Henn B. B.a. Coull, and R.O. Wright, Chemical mixtures and children's health. Curr Opin Pediatr. 2014;26(2):223-9.

32. Agency for Toxic Substances and Disease Registry (ATSDR). Toxicological profile for Selenium. Atlanta, GA: U.S. Department of Health and Human Services, Public Health Service. 2003.

33. Agency for Toxic Substances and Disease Registry (ATSDR). Toxicologica profile for Copper. Atlanta, GA: U.S. Department of Health and Human Services, Public Health Service. 2004.

34. Gerberding J. Toxicological profile for zinc. Atlanta: US Department of Health and Human Services, Agency for Toxic Substances and Disease Registry; 2005. p. 11-118.

35. Greger JL. Nutrition versus toxicology of manganese in humans: evaluation of potential biomarkers. Neurotoxicology. 1999;20(2-3):205-12.

36. Shenkin A. Dietary reference values for vitamin a, vitamin K, arsenic, boron, chromium, copper, iodine, iron, manganese, molybdenum, nickel, silicon, vanadium and zinc. J Hum Nutr Diet. 2003;16(3):199-200.

37. Yilmaz B, et al. Endocrine disrupting chemicals: exposure, effects on human health, mechanism of action, models for testing and strategies for prevention. In: Reviews in endocrine and metabolic disorders; 2019. p. 1-21.

38. Bloom MS, et al. Toxic trace metals and human oocytes during in vitro fertilization (IVF). Reprod Toxicol. 2010;29(3):298-305.

39. Deb P, Mandal SS. Endocrine disruptors: mechanism of action and impacts on health and environment. In: Gene regulation, epigenetics and hormone signaling; 2017. p. 607-38.

40. Wallace DR. Metals as endocrine disruptors in the environment. EC Pharmacol Toxicol ECO. 2019;2:12-4.

41. Mendiola J, et al. Relationships between heavy metal concentrations in three different body fluids and male reproductive parameters: a pilot study. Environ Health. 2011:10(1):6.

42. De Coster S, van Larebeke N. Endocrine-disrupting chemicals: associated disorders and mechanisms of action. J Environ Public Health. 2012:2012:713696.

43. Lauretta $R$, et al. Endocrine disrupting chemicals: effects on endocrine glands. Front Endocrinol (Lausanne). 2019;10:178.

44. lavicoli I, Fontana L, Bergamaschi A. The effects of metals as endocrine disruptors. J Toxicol Environ Health B Crit Rev. 2009;12(3):206-23.

45. Zeng $X$, et al. Alterations of serum hormone levels in male workers occupationally exposed to cadmium. J Toxicol Environ Health A. 2002;65(7):513-21.

46. Jurasovic J, et al. Semen quality and reproductive endocrine function with regard to blood cadmium in Croatian male subjects. Biometals. 2004;17(6):735-43.

47. Zeng $X$, et al. Impact of cadmium exposure on male sex hormones: a population-based study in China. Environ Res. 2004;96(3):338-44.

48. Telisman $\mathrm{S}$, et al. Reproductive toxicity of low-level lead exposure in men Environ Res. 2007;105(2):256-66.

49. Menke $A$, et al. The association of urinary cadmium with sex steroid hormone concentrations in a general population sample of US adult men BMC Public Health. 2008:8:72

50. Meeker JD, et al. Environmental exposure to metals and male reproductive hormones: circulating testosterone is inversely associated with blood molybdenum. Fertil Steril. 2010;93(1):130-40. 
51. Nagata C, et al. Urinary cadmium and serum levels of estrogens and androgens in postmenopausal Japanese women. Cancer Epidemiol Biomarkers Prev. 2005;14(3):705-8.

52. Garcia-Morales $P$, et al. Effect of cadmium on estrogen receptor levels and estrogen-induced responses in human breast cancer cells. J Biol Chem. 1994;269(24):16896-901.

53. Bochud $M$, et al. Urinary cadmium excretion is associated with increased synthesis of cortico-and sex steroids in a family-based Swiss population study. In: 19th European congress of endocrinology: BioScientifica; 2017.

54. Hauser R, et al. Association of blood lead levels with onset of puberty in Russian boys. Environ Health Perspect. 2008;116(7):976-80.

55. Williams PL, et al. Blood lead levels and delayed onset of puberty in a longitudinal study of Russian boys. Pediatrics. 2010;125(5):e1088-96.

56. Williams PL, et al. Blood lead levels and timing of male sexual maturity: a longitudinal study of Russian boys. Environ Int. 2019:125:470-7.

57. Agusa T, et al. Mercury in hair and blood from residents of Phnom Penh (Cambodia) and possible effect on serum hormone levels. Chemosphere. 2007;68(3):590-6.

58. Gerhard I, et al. Impact of heavy metals on hormonal and immunological factors in women with repeated miscarriages. Hum Reprod Update. 1998; 4(3):301-9.

59. Hsieh Fl, et al. Risk of erectile dysfunction induced by arsenic exposure through well water consumption in Taiwan. Environ Health Perspect. 2008; 116(4):532-6.

60. Meeker JD, et al. Cadmium, lead, and other metals in relation to semen quality: human evidence for molybdenum as a male reproductive toxicant. Environ Health Perspect. 2008;116(11):1473-9.

61. Telisman $\mathrm{S}$, et al. Semen quality and reproductive endocrine function in relation to biomarkers of lead, cadmium, zinc, and copper in men. Environ Health Perspect. 2000;108(1):45-53.

62. Jeng HA, et al. Role of low exposure to metals as male reproductive toxicants. Int J Environ Health Res. 2015;25(4):405-17.

63. Liu $Y$, et al. Fluoride exposure and pubertal development in children living in Mexico City. Environ Health. 2019;18(1):26.

64. Cahill L. Why sex matters for neuroscience. Nat Rev Neurosci. 2006;7(6):477-84.

65. Spear LP. The adolescent brain and age-related behavioral manifestations. Neurosci Biobehav Rev. 2000;24(4):417-63.

66. Parent $\mathrm{AS}$, et al. Developmental variations in environmental influences including endocrine disruptors on pubertal timing and neuroendocrine control: revision of human observations and mechanistic insight from rodents. Front Neuroendocrinol. 2015;38:12-36.

67. Perng W, et al. Early life exposure in Mexico to ENvironmental toxicants (ELEMENT) project. BMJ Open. 2019;9(8):e030427.

68. Wu Y, et al. Association of blood leukocyte DNA methylation at LINE-1 and growth-related candidate genes with pubertal onset and progression. Epigenetics. 2018;13(12):1222-33.

69. Lewis $\mathrm{RC}$, et al. Predictors of urinary bisphenol a and phthalate metabolite concentrations in Mexican children. Chemosphere. 2013;93(10):2390-8.

70. Ashrap $P$, et al. In utero and peripubertal metals exposure in relation to reproductive hormones and sexual maturation and progression among girls in Mexico City. Environ Res. 2019;177:108630.

71. Basu N, et al. A combined ecological and epidemiologic investigation of metal exposures amongst indigenous peoples near the Marlin mine in Western Guatemala. Sci Total Environ. 2010;409(1):70-7.

72. Srigboh RK, et al. Multiple elemental exposures amongst workers at the Agbogbloshie electronic waste (e-waste) site in Ghana. Chemosphere. 2016;164:68-74.

73. Lewis RC, et al. Urinary metal concentrations among mothers and children in a Mexico City birth cohort study. Int J Hyg Environ Health. 2018;221(4):609-15.

74. Chavarro JE, et al. Validity of self-assessed sexual maturation against physician assessments and hormone levels. J Pediatr. 2017;186:172-8 e3.

75. Marshall WA, Tanner JM. Variations in pattern of pubertal changes in girls. Arch Dis Child. 1969:44(235):291-303.

76. Mouritsen A, et al. The pubertal transition in 179 healthy Danish children: associations between pubarche, adrenarche, gonadarche, and body composition. Eur J Endocrinol. 2013;168(2):129-36.

77. Ankarberg-Lindgren C, Norjavaara E. Changes of diurnal rhythm and levels of total and free testosterone secretion from pre to late puberty in boys: testis size of $3 \mathrm{ml}$ is a transition stage to puberty. Eur J Endocrinol. 2004; 151(6):747-57.

78. Sergeyev $\mathrm{O}$, et al. The association of peripubertal serum concentrations of organochlorine chemicals and blood lead with growth and pubertal development in a longitudinal cohort of boys: a review of published results from the Russian Children's study. Rev Environ Health. 2017; 32(1-2):83-92.

79. AMAl. Avances del Comité de Niveles Socioeconómicos. En: Comité de Niveles Socioeconómicos. México, D. F.: Asociación Mexicana de Agencias de Investigación de Mercados y Opinión Pública, AC; 2004. cited; Available from: http://www.amai.org/niveles.php.

80. López, H. "Nivel socioeconómico AMAl." La Asociación Mexicana de Agencias de Investigación de Mercado (AMAI), editor. National Institute of Statistics and Geography. 2008.

81. Sitlani $\mathrm{CM}$, et al. Generalized estimating equations for genome-wide association studies using longitudinal phenotype data. Stat Med. 2015;34(1):118-30.

82. Sullivan Pepe M, Anderson GL. A cautionary note on inference for marginal regression models with longitudinal data and general correlated response data. Commun Stat-Simul Comput. 1994;23(4):939-51.

83. Peduzzi $P$, et al. A simulation study of the number of events per variable in logistic regression analysis. J Clin Epidemiol. 1996;49(12):1373-9.

84. Vittinghoff $E$, McCulloch CE. Relaxing the rule of ten events per variable in logistic and cox regression. Am J Epidemiol. 2007;165(6):710-8.

85. Benjamini $Y$, Hochberg $Y$. Controlling the false discovery rate: a practical and powerful approach to multiple testing. J R Stat Soc Ser B Methodol. 1995;57(1):289-300.

86. Ferguson KK, et al. Prenatal and peripubertal phthalates and bisphenol a in relation to sex hormones and puberty in boys. Reprod Toxicol. 2014;47:70-6.

87. Siu ER, et al. Cadmium-induced testicular injury. Toxicol Appl Pharmacol. 2009;238(3):240-9.

88. Liu J, Qu W, Kadiiska MB. Role of oxidative stress in cadmium toxicity and carcinogenesis. Toxicol Appl Pharmacol. 2009;238(3):209-14.

89. Patra RC, Amiya KR, Swarup D. "Oxidative stress in lead and cadmium toxicity and its amelioration." Vet Med Int. 2011;2011:457327.

90. Gay F, Vincenza L, Ivana C, Carla E, Marilena L, Anna C. "Chronic exposure to cadmium disrupts the adrenal gland activity of the newt Triturus carnifex (Amphibia, Urodela)." Biomed Res Int. 2013;2013:424358.

91. Singhal R, Merali Z, Hrdina P. Aspects of the biochemical toxicology of cadmium. In: Federation proceedings; 1976.

92. Schwarz G. Molybdenum cofactor and human disease. Curr Opin Chem Biol 2016;31:179-87.

93. Todd GD, et al. Toxicological profile for molybdenum: draft for public comment; 2017.

94. Lewis RC, Meeker JD. Biomarkers of exposure to molybdenum and other metals in relation to testosterone among men from the United States National Health and nutrition examination survey 2011-2012. Fertil Steril. 2015;103(1):172-8

95. Thomas J, Moss S. The effect of orally administered molybdenum on growth, spermatogenesis and testes histology of young dairy bulls. J Dairy Sci. 1951;34(9):929-34.

96. Jeter MA, Davis GK. The effect of dietary molybdenum upon growth, hemoglobin, reproduction and lactation of rats. J Nutr. 1954;54(2):215-20.

97. Vyskočil A, Viau C. Assessment of molybdenum toxicity in humans. J Appl Toxicol. 1999;19(3):185-92.

98. Pandey R, Singh S. Effects of molybdenum on fertility of male rats. Biometals. 2002;15(1):65-72.

99. Centers for Disease Control and Prevention. Fourth report on human exposure to environmental chemicals; 2018. [04/04/2018]; Available from: https://www.cdc.gov/exposurereport/pdf/FourthReport_UpdatedTables_ Volume1_Mar2018.pdf.

100. Kwon B, et al. Effects of barium chloride exposure on hormones and genes of the hypothalamic-pituitary-gonad Axis, and reproduction of Zebrafish (Danio rerio). Bull Environ Contam Toxicol. 2016;96(3):341-6.

101. Leonard MB, et al. Plasma zinc status, growth, and maturation in children with sickle cell disease. J Pediatr. 1998;132(3 Pt 1):467-71.

102. Wagner PA, et al. Serum zinc concentrations in adolescents as related to sexual maturation. Hum Nutr Clin Nutr. 1985;39(6):459-62.

103. Vivoli $G$, et al. Relationship between zinc in serum and hair and some hormones during sexual maturation in humans. Sci Total Environ. 1990;95: 29-40.

104. Onukwuli VO, et al. Impact of zinc on sexual maturation of female sickle cell anemia (SCA) children in Enugu, Southeast Nigeria. Pediatr Hematol Oncol. 2018;35(2):145-55.

105. Kerns K, Michal Z, Peter S. "Zinc: A necessary ion for mammalian sperm fertilization competency." Int J Mol Sci. 2018;19(12):4097. 
106. Mendoza AD, et al. Zinc availability during germline development impacts embryo viability in Caenorhabditis elegans. Comp Biochem Physiol C Toxicol Pharmacol. 2017;191:194-202.

107. Arangasamy A, et al. Advancement of puberty and enhancement of seminal characteristics by supplementation of trace minerals to bucks. Theriogenology. 2018;110:182-91.

108. Geary T, et al. Effect of supplemental trace mineral level and form on peripubertal bulls. Anim Reprod Sci. 2016;168:1-9.

109. Dance A, et al. Enhanced early-life nutrition of Holstein bulls increases sperm production potential without decreasing postpubertal semen quality. Theriogenology. 2016;86(3):687-694. e2.

110. El-Masry KA, Nasr AS, Kamal TH. Influences of season and dietary supplementation with selenium and vitamin E or zinc on some blood constituents and semen quality of New Zealand white rabbit males. World Rabbit Sci. 1994;2(3).

111. Fang VS, Furuhashi N. Partial alleviation of the antitesticular effect of pipecolinomethylhydroxyindane by zinc in rats. J Endocrinol. 1978;79(1): $151-2$.

112. Underwood E, Somers M. Studies of zinc nutrition in sheep. I. the relation of zinc to growth, testicular development, and spermatogenesis in young rams. Aust J Agr Res. 1969;20(5):889-97.

113. Breehl L, Caban O. Physiology, puberty. Treasure Island: StatPearls; 2020 .

114. Rubinow DR, Schmidt PJ. Androgens, brain, and behavior. Am J Psychiatry. 1996;153(8):974-84.

115. German A, et al. Outcomes of pubertal development in girls as a function of pubertal onset age. Eur J Endocrinol. 2018;179(5):279-85.

116. Pantsiotou S, et al. Maturational tempo differences in relation to the timing of the onset of puberty in girls. Acta Paediatr. 2008;97(2):217-20.

117. Llop-Viñolas D, et al. Onset of puberty at eight years of age in girls determines a specific tempo of puberty but does not affect adult height. Acta Paediatr. 2004:93(7):874-9.

\section{Publisher's Note}

Springer Nature remains neutral with regard to jurisdictional claims in published maps and institutional affiliations.

Ready to submit your research? Choose BMC and benefit from:

- fast, convenient online submission

- thorough peer review by experienced researchers in your field

- rapid publication on acceptance

- support for research data, including large and complex data types

- gold Open Access which fosters wider collaboration and increased citations

- maximum visibility for your research: over $100 \mathrm{M}$ website views per year

At $\mathrm{BMC}$, research is always in progress.

Learn more biomedcentral.com/submissions 